https://doi.org/10.22319/rmcp.v11i1.4674

Nota de investigación

\title{
Dinámica de crecimiento y curvas de extracción de nutrientes de Pennisetum sp. (Maralfalfa)
}

Oscar López-Astilleros ${ }^{\text {a }}$

Julio Cesar Vinay Vadillo ${ }^{b}$

Yuri Villegas-Aparicio ${ }^{\text {a }}$

Isaías López Guerrero b

Salvador Lozano-Trejo ${ }^{a^{*}}$

a Instituto Tecnológico del Valle de Oaxaca, División de Estudios de Posgrado e Investigación. Programa de Maestría en Ciencias, Oaxaca México.

b CIR Golfo Centro/INIFAP/SAGARPA. Campo experimental "La Posta", Carretera La Tinaja-Paso del Toro, 94270 Medellín, Veracruz-Llave, México.

*Autor de correspondencia: lozanos2004@gmail.com

\section{Resumen:}

El crecimiento de una especie, así como la concentración y extracción de nutrientes en el forraje, se pueden conocer a través de modelos matemáticos. Se utilizaron las ecuaciones de Gompertz y Logistic para estudiar el crecimiento, un modelo potencial negativo para las curvas de dilución NPK y la extracción máxima a través de polinomios de segundo orden. Se calculó la Extracción Unitaria (EU) y Eficiencia de Recuperación del Nutriente (ERN) NPK. La bondad de ajuste de los modelos fue comparada bajo un diseño completamente al azar con arreglo factorial $2 \times 2$, factor A: dos modelos y factor B: fertilización y testigo con 16 repeticiones. La concentración de nutrientes se optimizó por algoritmo de LevenbergMarquart. Los resultados indicaron que el ajuste del modelo fue similar para las parcelas en estudio; sin embargo, Gompertz mostró una mejor representación de la realidad biológica. La máxima tasa de crecimiento se alcanzó a los 21 días en parcelas testigo y 56 días en 
parcelas fertilizadas, el punto de inflexión a los 30 y 31 días, respectivamente. La concentración de nutrientes en el pasto, disminuyó a través de los cortes en ambos tratamientos y las parcelas fertilizadas además presentaron la mayor producción de materia seca. La EU se mostró casi de manera homogénea para parcelas fertilizadas y no fertilizadas. La ERN fue de $48 \%$ para nitrógeno, $39 \%$ para fósforo y $104 \%$ para potasio, lo cual sugiere un suministro excedido de $\mathrm{N}$ y $\mathrm{P}$ con la dosis suministrada y un consumo de lujo para $\mathrm{K}$.

Palabras clave: Dilución de nutrientes, Gompertz, Logistic, Tasa de crecimiento.

Recibido: $23 / 10 / 2017$

Aceptado:14/12/2018

La producción continua de forraje es la meta más apremiante para satisfacer las necesidades de alimentación en los sistemas de producción bovina de carne y leche ${ }^{(1)}$. La ganadería tropical que desarrollan pequeños y medianos productores, enfrenta la escasez de forraje generada por sequías e inadecuadas prácticas de manejo ${ }^{(2,3)}$. Las forrajeras de corte que se cultivan en tierras con aptitud productiva, son fundamentales para reducir los costos de producción en la alimentación del ganado $^{(4)}$, con menos superficie necesaria para la producción de forraje y menor impacto sobre los ecosistemas transformados por un creciente desarrollo ganadero intensivo ${ }^{(3)}$. Ante los retos de una ganadería sustentable, cualquier especie que se precie de ser productiva, es necesario conocer su velocidad de crecimiento y obtener parámetros de referencia que conduzcan a la toma de decisiones de manejo y aumentar la eficiencia en el aprovechamiento del cultivo ${ }^{(5,6)}$, asimismo garantizar la expresión de su máximo potencial productivo.

Los Pennisetum spp. pueden ser implementados en sistemas intensivos de corte, ya que presentan rápido crecimiento y alto volumen de producción ${ }^{(7,8)}$. Por consiguiente, es importante retomar el uso de modelos matemáticos que permitan determinar el momento de máxima producción biológica del cultivo, así como de la extracción de nutrientes NPK; lo que ayudaría a planificar el momento de aprovechamiento del forraje, suministro de fertilizantes y evitar un mayor impacto ambiental al sistema natural.

La obtención de mejores rendimientos de forraje se ha asociado al aporte de nutrientes en el suelo, los cuales contribuyen a mejorar el valor nutritivo y producción del cultivo ${ }^{(9)}$. Adicionalmente, la acumulación de unidades calor debe ser suficiente y con adecuado aporte de agua para aprovechar el potencial productivo de la especie forrajera ${ }^{(10)}$. 
La presente investigación tuvo como primer objetivo describir el crecimiento de Pennisetum sp. (Maralfalfa) con fertilización y testigo en la época lluviosa, a través de los modelos Gompertz y Logistic. Además, modelar las curvas de extracción y dilución de nutrientes, calcular la EU y la ERN de nitrógeno $(\mathrm{N})$, fósforo $(\mathrm{P})$ y potasio $(\mathrm{K})$, para determinar la dosis del siguiente ciclo productivo.

El establecimiento del pasto maralfalfa se llevó a cabo bajo condiciones de temporal en julio de 2010 a enero de 2011, en el campo experimental "La Posta", ubicado en Paso del Toro, Veracruz coordenadas $19^{\circ} 00^{\prime} 49^{\prime \prime} \mathrm{N}$, y $96^{\circ} 08^{\prime} 19^{\prime \prime} \mathrm{O}$, a $10 \mathrm{msnm}^{(11)}$. El clima predominante corresponde al $\mathrm{Aw}_{2}$ tropical sub-húmedo de acuerdo a la clasificación climática de Köppen $^{(12)}$, con una precipitación acumulada durante el estudio de $1,461 \mathrm{~mm}$ y humedad relativa promedio de $77.4 \%$; la temperatura máxima, media y mínima de 35,25 y $15{ }^{\circ} \mathrm{C}$, respectivamente; suelos predominante tipo Vertisol profundos con $\mathrm{pH}$ ácido (5.4), textura migajón arcillo-arenosa y contenido de materia orgánica de $2.6 \%{ }^{(13)}$. La unidad experimental fue de 4.0 x $12.0 \mathrm{~m}$, sembradas en surcos a $0.80 \mathrm{~m}$ de distancia y $0.80 \mathrm{~m}$ entre plantas, bajo un diseño en bloques completos al azar y 16 repeticiones. La dosis de fertilización suministrada fue 141-43-20 (N-P-K), utilizando $200 \mathrm{~kg}$ de urea, $50 \mathrm{~kg}$ de la mezcla 18-46-00 y $200 \mathrm{~kg}$ de la mezcla 20-10-10; en dos aplicaciones, la primera con $100 \mathrm{~kg}$ de urea, $100 \mathrm{~kg}$ de la mezcla $20-10-10$ y $25 \mathrm{~kg}$ de la mezcla $18-46-00$ ocho días después del corte de uniformidad. A los sesenta días posteriores, se aplicó el resto del nitrógeno, fósforo y potasio ${ }^{(14)}$.

El pasto se cosechó cada 21 días a $25 \mathrm{~cm}$ del suelo, con ocho cortes hasta 168 días. En cada muestreo se registró la producción de materia verde (MV) por parcela, y del material fresco se colectó una sub-muestra de $300 \mathrm{~g}$, la cual se secó en estufa de aire forzado a una temperatura de $55^{\circ} \mathrm{C}$ hasta peso constante, para estimar el contenido de materia seca (MS) con corrección a $105{ }^{\circ} \mathrm{C}^{(15)}$, las muestras se molieron en molino Thomas Wiley, modelo 3383L40 a $1 \mathrm{~mm}$ de tamaño para los análisis químicos NPK. Con los pesos acumulados de MS se obtuvo la curva y tasa de crecimiento del cultivo mediante la ecuación 1.

$$
T C=\frac{M S P_{t 2}-M S P_{t 1}}{T 2-T 1}
$$

Donde: $\mathrm{TC}=$ tasa de crecimiento $\mathrm{kg} \mathrm{MS} \mathrm{ha} \mathrm{d}^{-1} \mathrm{~d}^{-1}, \mathrm{MSP}_{\mathrm{t} 2}=$ materia seca presente en el tiempo $2, \mathrm{~kg} \mathrm{MS} \mathrm{ha}^{-1}, \mathrm{MSP}_{\mathrm{t} 1}=$ materia seca presente en el tiempo $1, \mathrm{~kg} \mathrm{MS} \mathrm{ha}^{-1}$ $\mathrm{T} 2=$ tiempo final, $\mathrm{T} 1=$ tiempo inicial.

Las curvas de crecimiento se obtuvieron con los modelos Gompertz ${ }^{(16)}$ y Logistic ${ }^{(17)}$, se utilizó el software Micromath Scientist ${ }^{\circledR}$ (Micromath Research, 2006), aplicando el algoritmo de Powell como método de minimización. La función Gompertz utilizada fue: 


$$
Y=A \exp ^{-\exp (-\mu(x-B))}
$$

Donde $\mathrm{Y}=$ rendimiento, $A=$ máxima producción, $-B=$ punto de inflexión, $\mu=$ velocidad del crecimiento y $x=$ tiempo en días. La función Logistic utilizada fue:

$$
P=A /\left(1+B^{*} \exp \left(-c^{*} T\right)\right)
$$

Donde $\mathrm{P}=$ rendimiento, $\mathrm{A}=$ máxima producción, $\mathrm{B}=$ punto de inflexión y $c=$ velocidad de crecimiento $^{(18)}$.

El contenido de nitrógeno en el forraje se determinó por el método Kjeldhal (VELP Scientifica, Series D-K6, USA), en muestras de $0.5 \mathrm{~g}$ por triplicado de planta completa; fósforo con vanadato de amonio en espectrofotómetro UV-Visible (UV/VIS Lambda 2, Perkin Elmer, USA). Potasio, calcio y magnesio por absorción atómica y azufre por el método turbidimétrico con sulfato de bario en espectrofotómetro UV-Visible ${ }^{(19)}$. La composición nutrimental NPK en las muestras de forraje a través de los cortes, se determinó mediante la ecuación $4^{(20)}$.

$$
y=a x^{-B}
$$

Donde $a=$ concentración crítica de nutriente en la planta, $x=$ es la producción de MS, $-B=$ la tasa de disminución (dilución) del nutriente en la planta, utilizando el algoritmo de Levenberg-Marquart, para minimización de la varianza.

La extracción unitaria (EU) de cada nutriente, se determinó empleando la composición nutrimental (N, P, K) en la especie evaluada con la ecuación $5^{(21)}$.

$\mathrm{EU}=\mathrm{RG} / \mathrm{NAF} \quad(5)$

Donde: $\mathrm{EU}=$ extracción unitaria o eficiencia de uso de nutrimento en el fertilizante, $\mathrm{RG}=$ rendimiento de forraje $\left(\mathrm{kg} \mathrm{ha}^{-1}\right)$, y NAF= cantidad del nutrimento aplicado proveniente del fertilizante $\left(\mathrm{kg} \mathrm{ha}^{-1}\right)$.

Para calcular la eficiencia de recuperación del nutriente (ERN) NPK, se utilizó la siguiente fórmula ${ }^{(22)}$.

$\mathrm{ERN}=[(\mathrm{NT}-\mathrm{Ntestigo}) /$ Dosis en el trat $] \times 100$ (6)

Donde: $\mathrm{ERN}=$ eficiencia de recuperación del nutriente, $\mathrm{NT}=$ contenido del nutriente en planta en el tratamiento (NPK); Ntestigo= contenido del nutriente en planta en el testigo y dosis en el trat $=$ dosis del nutriente aplicado en el fertilizante (NPK). NT y NTestigo se obtuvieron a través de la derivada de cada polinomio ajustado, para la extracción máxima de nutrientes NPK. 
Los indicadores de bondad de ajuste de los modelos Gompertz y Logístic utilizados fueron: $\mathrm{R}^{2}, \mathrm{R}^{2}$ ajustado, $\mathrm{r}^{(23)}$ y el criterio de selección del modelo (MSC; por sus siglas en inglés) ${ }^{(24)}$. A los coeficientes A, B, C y $\mu$; ERN se les aplicó un análisis de varianza con el procedimiento GLM (SAS ver. 9.01) y una comparación de medias por el método de Duncan $(\alpha=0.05)^{(25)}$.

El modelo Gompertz mostró una mejor representación de la realidad biológica en el punto de inflexión (B) para el pasto fertilizado y testigo. El rendimiento máximo de MS fue de $10,414 \mathrm{~kg} \mathrm{MS} \mathrm{ha}^{-1}$ en parcelas con fertilización a los 161 días y de $5,952 \mathrm{~kg} \mathrm{MS} \mathrm{ha}^{-1}$ en parcelas testigo a los 168 días, (Cuadro 1). La tasa de crecimiento se mantuvo en ascenso en las parcelas con fertilización hasta los 56 días, con punto de inflexión a los 31 días (Figura 1a). En parcelas testigo, la máxima tasa de crecimiento se presentó a los 21 días para después mostrar tasas descendentes y el punto de inflexión a los 30 días (Figura 1b). Los resultados indicaron que el comportamiento de crecimiento del pasto maralfalfa fue similar a otras especies tropicales ${ }^{(26)}$, con una curva sigmoidea hasta alcanzar su máxima expresión y después decrecer en forma asintótica, lo que indica un ajuste razonable de los datos por el modelo Gompertz y una realidad biológica observada en la curva; obteniendo parámetros confiables que proporcionan información sobre las características de crecimiento ${ }^{(18)}$.

Cuadro 1: Indicadores de bondad de ajuste y coeficientes del modelo Gompertz

\begin{tabular}{llc}
\hline Indicadores/coeficientes & $\begin{array}{c}\text { Maralfalfa } \\
\text { Fertilizado }\end{array}$ & $\begin{array}{c}\text { Maralfalfa } \\
\text { no fertilizado }\end{array}$ \\
\hline $\mathrm{R}^{2}$ & $0.98 \pm 0.004 \mathrm{a}$ & $0.98 \pm 0.004 \mathrm{a}$ \\
$\mathrm{R}^{2} \mathrm{aj}$ & $0.87 \pm 0.028 \mathrm{a}$ & $0.84 \pm 0.046 \mathrm{a}$ \\
$\mathrm{r}$ & $0.93 \pm 0.015 \mathrm{a}$ & $0.92 \pm 0.025 \mathrm{a}$ \\
$\mathrm{MSC}$ & $1.54 \pm 0.262 \mathrm{a}$ & $1.44 \pm 0.378 \mathrm{a}$ \\
& & \\
A máxima producción (kg ha & & \\
$\mathrm{B}$ punto de inflexión (días) & $10,414 \pm 2,254.57 \mathrm{a}$ & $5,952 \pm 2,684.75 \mathrm{~b}$ \\
$\mu$ velocidad de crecimiento (kg MS ha ${ }^{-1}$ día $^{-}$ & $31.12 \pm 7.094 \mathrm{a}$ & $29.62 \pm 15.946 \mathrm{a}$ \\
1) & $43 \pm 0.68 \mathrm{a}$ & $31 \pm 0.54 \mathrm{~b}$ \\
\hline
\end{tabular}

ab Letras diferentes entre columnas con significancia estadística (Duncan= 0.05 ). acompañadas \pm error estándar.

$\mathrm{R}^{2}=$ coeficiente de determinación, $\mathrm{R}^{2} \mathrm{aj}=$ coeficiente de determinación ajustado. $\mathrm{r}=$ coeficiente de correlación, $\mathrm{MSC}=$ criterio de selección del modelo. 
Figura 1: Curva y tasa de crecimiento ajustados (Gompertz), ecotipo maralfalfa con fertilización (a) y testigo (b) en época de lluvias

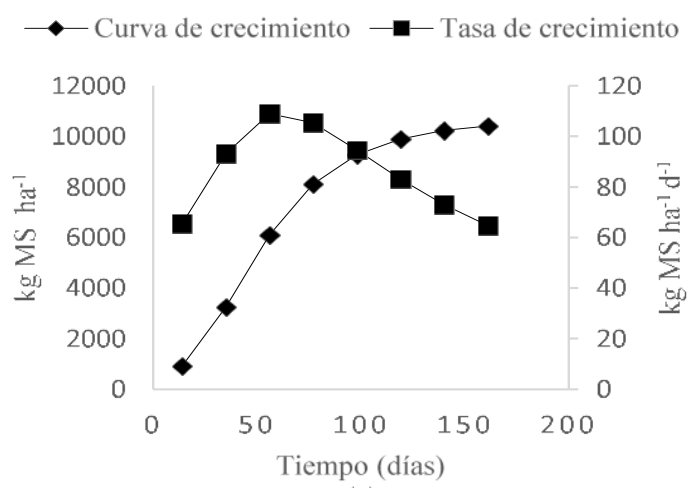

(a)

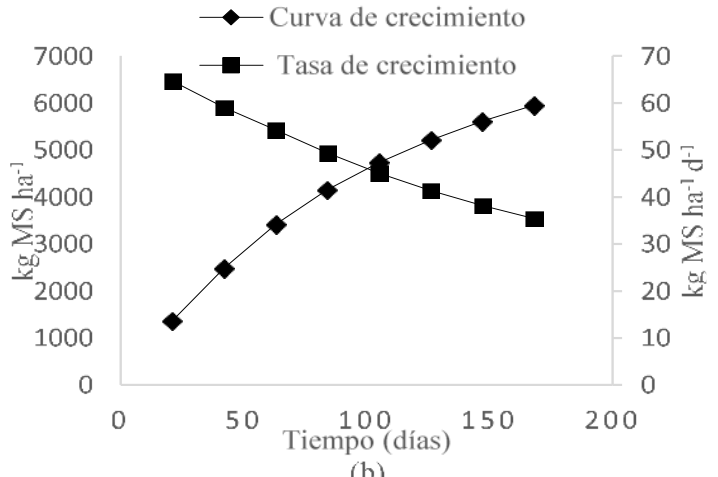

(b)

Los bajos rendimientos de forraje en el tratamiento testigo, se relacionan con la baja disponibilidad de nutrientes en el suelo ${ }^{(27)}$. En variedades de Pennisetum OM-22 fertilizadas a los 56 días, se han obtenido rendimientos de $7 \mathrm{t} \mathrm{MS} \mathrm{ha}{ }^{-1(23)}$, superior a las $6.1 \mathrm{t} \mathrm{ha}^{-1}$ producidas en este estudio a los 56 días al corte; que es el máximo potencial productivo expresado, de acuerdo a los procesos fisiológicos de fotosíntesis, acumulación de unidades calor, absorción de agua, disponibilidad de nutrimentos y crecimiento según Colabelli et $a l^{(10)}$. De esta forma, se aprovecha de manera óptima el forraje, evitando las pérdidas de biomasa total por senescencia y descomposición ${ }^{(9)}$.

La concentración de nutrientes NPK presentó promedios de 1.06, 0.20 y $2.35 \%$ vs 0.79, 0.21, $2.30 \%$ en parcelas con fertilización y testigo $(P>0.05)$, respectivamente. No obstante, la mayor disponibilidad de nutrientes en el suelo por efecto de la fertilización se mantuvo por más tiempo, lo cual produjo mayor MS acumulada. La tasa de crecimiento ( $\mu$ en la Ecuación 2) fue mayor para el pasto fertilizado; por lo tanto, los rendimientos son mayores por unidad de tiempo y espacio como ha sido demostrado en diversos estudios de otros cultivos y forrajes, con mejores niveles de proteína ${ }^{(5,28,29)}$. Sin embargo, el momento de la máxima producción y el más alto valor nutritivo del forraje no es concordante, situación que debe ser determinada por el productor cortando el forraje entre los 30 y 56 días, para cosechar un pasto de mejor calidad y aprovechar la máxima producción de maralfalfa.

En varios estudios se ha reportado que el contenido de nitrógeno disminuye en la planta con el transcurso del tiempo ${ }^{(29,30)}$, esto es después de los 30 días de crecimiento; por lo que su calidad nutricional disminuye, ya que con la edad se experimentan disminuciones graduales en su composición química ${ }^{(29,30)}$; lo cual ocurre también con fósforo y potasio.

Las máximas extracciones de NPK en las parcelas testigo, se dieron a los 107, 110 y 120 días, con 30, 10 y $110 \mathrm{~kg} \mathrm{ha}^{-1}$, respectivamente. En las parcelas con fertilización, la máxima 
extracción se presentó a los 98 días para $\mathrm{N}$, a los 115 días para fósforo y a los 116 días para potasio; con una extracción máxima de 68, 17 y $208 \mathrm{~kg} \mathrm{ha}^{-1}$ de NPK, respectivamente (Figuras 2a y 2b). La extracción unitaria fue similar en ambos tratamientos (con y sin fertilización); por lo que la extracción total promedio fue de $27.49 \mathrm{~kg}$ de N, $10.05 \mathrm{~kg}$ de P y $116.0 \mathrm{~kg}$ de $\mathrm{K}$ para las $5.952 \mathrm{t} \mathrm{MS} \mathrm{ha}^{-1}$ promedio, producidas en los 168 días del cultivo en parcelas testigo. Con fertilización se estimó una extracción unitaria de $4.72 \mathrm{~kg} \mathrm{~N}, 1.29 \mathrm{~kg} \mathrm{P}$, y $15.90 \mathrm{~kg}$ de K por tonelada de MS, donde la extracción total promedio fue de $49.15 \mathrm{~kg}$ de $\mathrm{N}, 13.43 \mathrm{~kg}$ de $\mathrm{P}$ y $165.58 \mathrm{~kg}$ de K para una producción máxima de $10.414 \mathrm{t} \mathrm{MS} \mathrm{ha}{ }^{-1}$ a los 161 días del cultivo. Los resultados de la ERN de NPK 48, 39 y $104 \%$, respectivamente, permitieron conocer la cantidad de nutriente requerido por maralfalfa en el periodo de estudio, con una producción acumulada de 58. 205 t ha-1 a los 161 días; lo cual, bajo el planteamiento de Volke et $a l^{(31)}$ en el forraje fertilizado nos permite generar una recomendación de ajuste de fertilizante para el siguiente ciclo, consistente en 45-09-48 kg $\mathrm{ha}^{-1}$ de NPK, respectivamente.

Figura 2: Curvas de extracción de NPK (modelo cuadrático), del ecotipo maralfalfa con fertilización (a) y testigo (b) en época de lluvias

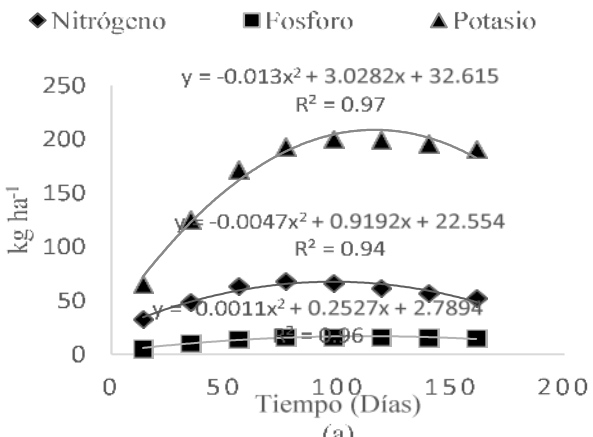

(a)

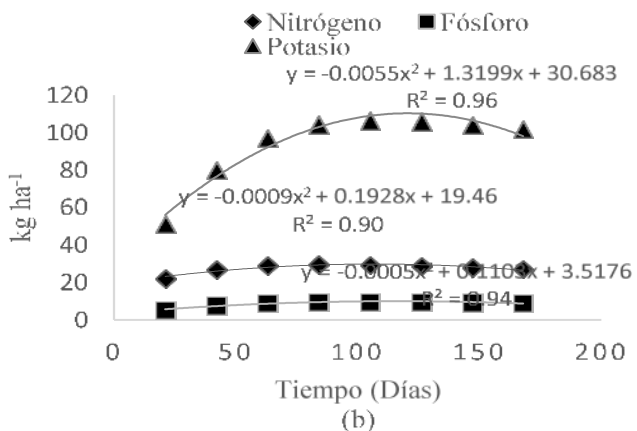

(b)

Se ha reportado en diversos estudios ${ }^{(32,33)}$ que un suministro excedido de $\mathrm{N}$ y $\mathrm{K}$ vía fertilización, disminuye la ERN de los mismos, sugiriendo una pérdida y contaminación del suelo por $\mathrm{N}$, y un consumo de lujo de $\mathrm{K}^{(33)}$; lo cual es una situación frecuente que enfrentan los productores de forraje. Por lo tanto, es necesario ajustar la dosis de fertilización basados en el balance nutrimental entre la demanda del nutrimento por el cultivo, el suministro del nutrimento por el suelo y la eficiencia de recuperación del nutriente ${ }^{(31)}$. La fertilización mostró efectos directos en la producción de MS y contenido NPK en el forraje. Sin embargo, la ERN fue menor para $\mathrm{N}$ y $\mathrm{P}$ lo cual sugiere un suministro excedido de estos nutrientes, a través del fertilizante. 
Los resultados muestran evidencias que el ecotipo maralfalfa, aprovecha el suministro de nutrientes vía fertilización en las condiciones climáticas locales (temporada lluviosa) para incrementar su rendimiento, duplicando la tasa de crecimiento en los primeros 60 días. Por lo que sería recomendable realizar el primer corte antes de los 56 días, momento en el que maralfalfa alcanza su máxima tasa de crecimiento y así aprovechar una mejor calidad del forraje. Si se desea una mayor concentración de NPK en el forraje cosechado, el corte debería realizarse entre los 30 y 35 días de rebrote en los cortes sucesivos. Los resultados también sugieren, que en el próximo ciclo productivo el suministro de fertilizante debería ajustarse a la baja. Adicionalmente, conocer el comportamiento productivo de maralfalfa respecto a la extracción de nutrientes, permitiría tomar decisiones de manejo del fertilizante, aumentando la eficiencia de uso de los nutrientes por el cultivo e indicando el momento oportuno para su suministro; a la vez podrían reducirse los excedentes potencialmente contaminantes, particularmente para $\mathrm{N}$.

\section{Literatura citada:}

1. Ramírez JL, Herrera RS, Leonard I, Cisneros M, Vardecia D, Álvarez Y. Relación entre factores climáticos, rendimiento y calidad de Pennisetum purpureum vc. Cuba CT 169 en el Valle del Cauto, Cuba. Rev Cubana Cien Agríc 2011;45(3):293-297.

2. Ortiz EE, Rodríguez JC, Russo RO. Efecto de fertilización y frecuencia de corte en rendimiento de biomasa de dos variedades del pasto king grass (Pennisetum purpureum). Tierra Tropical 2010;(6):43-53.

3. Steinfeld H, Gerber P, Wassenaar T, Castel V, Rosales M, De-Haan C (2009). La larga sombra del ganado: problemas ambientales y opciones. FAO. Roma.http://www.fao.org/3/a-a0701s.pdf. Consultado 7 Sep, 2017.

4. Márquez F, Sánchez J, Urbano D, Dávila C. Evaluación de la frecuencia de corte y tipos de fertilización sobre tres genotipos de pasto elefante (Pennisetum purpureum). 1. Rendimiento y contenido de proteína. Zootec Trop 2007;25(4): 253-259.

5. Rodríguez L, Larduet R, Martínez RO, Torres V, Herrera M, Medina Y, Noda AC. Modelación de la dinámica de acumulación de biomasa en Pennisetum purpureum vc. king grass en el occidente de Cuba. Rev Cubana Cien Agríc 2013;47(2):119-124.

6. Valenciaga D, Chongo B, Herrera RS, Torres V, Oramas A, Cairo JG, Herrera M. Efecto de la edad de rebrote en la composición química de Pennisetum purpureum vc. Cuba CT-115. Rev Cubana Cienc Agríc 2009;43(1)73-79. 
7. Calzada-Marín JM, Enríquez-Quiroz JF, Hernández-Garay A, Ortega-Jiménez E, Mendoza-Pedroza SI. Análisis de crecimiento del pasto Maralfalfa (Pennisetum sp.) en clima cálido subhúmedo. Rev Mex Cienc Pecu 2014;5(2):247-260.

8. Wagner B, Colón R. Comportamiento forrajero de tres Pennisetum purpureum Schumach. Revista APF 2014;3(1): 61-66.

9. De Caram GA, Angeloni P, Prause J. Determinación de la curva de dilución de nitrógeno en diferentes fases fenológicas del girasol. Agric Téc (Chile) 2007;67(2):189-195.

10. Colabelli MR, Agnusdei MG, Durand JL. Grupos funcionales de plantas, producción de forraje y eficiencia de uso de radiación de pastizales naturales en condiciones potenciales y limitadas de agua y nitrógeno. RIA (Argentina) 2011;(37):62-74.

11. INEGI (Instituto Nacional de Estadística y Geografía). Catálogo de claves de entidades federativas, municipios y localidades. 2009. http://mapserver. inegi. org. $\mathrm{mx} / \mathrm{mgn} 2 \mathrm{k} / \mathrm{s}=$ geo\&c=1223. Consultado 23 Jun, 2016.

12. Vidal ZR. Las Regiones Climáticas de México. Colección: Temas Selectos de Geografía de México. UNAM. México. 2005.

13. CIR Golfo Centro/INIFAP/SAGARPA. Campo experimental "La Posta". Caracterización y resultados de análisis de suelo en el Laboratorio de Suelos. Paso del Toro, Veracruz. 2011.

14. Ramos-Trejo OS, Victoria-Graniel CA, Sandoval-Gío JJ. Temporada, fertilización y rendimiento de variedades de Pennisetum purpureum. Agrociencia 2014;49(8):837-844.

15. AOAC. Official methods of analysis. Association of Official Analytical Chemists. 15th. ed, Washington DC, USA. 1980.

16. Raji AO, Alade NK, Duwa H. Estimation of model parameters of the japanese quail growth curve using Gompertz model. Arch Zootec 2014;63(243):429-435.

17. García- Cardozo CR, Martínez RO, Tuero R, Cruz AM, Estanquero L, Noda, AC, et al. Evaluación de Panicum maximum vc, Mombaza y modelación de indicadores agronómicos durante tres años en un suelo ferralítico rojo típico de la provincia la Habana. Rev Cubana Cien Agríc 2009;43(3):297-306.

18. Casas AG, Rodríguez D, Afanador TG. Propiedades matemáticas del modelo Gompertz y su aplicación al crecimiento de los cerdos. Rev Colomb Cienc Pecu 2010;(23):349358.

19. Rodríguez-Fuentes H, Rodriguez-Absi J. Métodos de análisis de suelo y plantas. México: Editorial Trillas SA de CV; 2011. 
20. Juárez-Hernández J, Bolaños-Aguilar ED. Las curvas de dilución de la proteína como alternativa para la evaluación de pastos tropicales. Uciencia 2007;23(1):81-90.

21. Castro-Luna I, Gavi-Reyes F, Peña-Cabriales JJ, Núñez-Escobar R, -Etchevers-Barra JD. Eficiencia de recuperación de $\mathrm{N}$ y $\mathrm{K}$ de tres fertilizantes de lenta liberación. Terra Latinoamericana 2006;24(2): 77-282.

22. Mora-Ravelo SG, Gavi-Reyes F, Tijerina-Chávez L, Pérez-Moreno J, Peña-Cabriales JJ. Evaluación de la recuperación del nitrógeno y fósforo de diferentes fuentes de fertilizantes por el cultivo de trigo irrigado con aguas residuales y de pozo. Acta Agronómica 2014;63(1):25-30.

23. Martínez RO, Tuero R, Torres V, Herrera RS. Modelos de acumulación de biomasa y calidad en las variedades de hierba elefante, Cuba CT-169, OM-22 y King Grass durante la estación lluviosa en el occidente de Cuba. Rev Cubana Cien Agríc 2010;44(2):189194.

24. Phaechamud T, Thongpin C, Choncheewa C. Shellac Wax-Lutrol F127 as Matrix Base for Hot Melt Extrusion. Res J Pharm Biol Chem Sci 2012;3(4):687-694. https://www.rjpbcs.com/pdf/2012_3(4)/[74].pdf

25. Steel RGD, Torrie JH. Bioestadística: principios y procedimientos. $2^{\mathrm{a}}$ ed en español. Editorial McGraw-Hill México ;1996.

26. Rodríguez L, Torres V, Martínez RO, Jay O, Noda AC, Herrera M. Modelos para estimar la dinámica de crecimiento de Pennisetum purpureum vc. Cuba CT-169. Rev Cubana Cien Agríc 2011;45(4):349-354.

27. Escudero A, Mediavilla S. Dinámica interna de los nutrientes. Ecosistemas 2003;(1):1-8.

28. Crespo G, Álvarez J. Comparación de la producción de biomasa de clones de Pennisetum purpureum fertilizados con nitrógeno. Rev Cubana Cien Agríc 2014;(3):287-291.

29. Cerdas-Ramírez R. Comportamiento productivo del pasto Maralfalfa (Pennisetum sp.) con varias dosis de fertilización nitrogenada. InterSedes 2015;16(33):124-145.

30. Uvidia H, Ramírez J, Vargas J, Leonard I, Sucoshañay J. Rendimiento y calidad del Pennisetum purpureum vc Maralfalfa en la Amazonía ecuatoriana. Red Vet 2015;16(6)1-11.

31. Volke HV, Etchevers BJD, San Juan RA, Silva PT. Modelo de balance nutrimental para la generación de recomendaciones de fertilización para cultivos. Terra Latinoamericana 1998;16(1):79-91. 
32. Cabalceta G, Saldias M, Alvarado A. Absorción de nutrimentos en el cultivar de papa MNF-80. Agronomía Costarricense 2005;29(3):107-123

33. Puentes-Páramo Y, Menjivar-Flores J, Aranzazu-Hernández F. Eficiencias en el uso de nitrógeno, fósforo y potasio en clones de cacao (Theobroma cacao L.). Bioagro 2014;26(2):99-106. 